\title{
Image Interpolation by Two-Dimensional Parametric Cubic Convolution
}

\author{
Jiazheng Shi and Stephen E. Reichenbach
}

\begin{abstract}
Cubic convolution is a popular method for image interpolation. Traditionally, the piecewise-cubic kernel has been derived in one dimension with one parameter and applied to two-dimensional (2-D) images in a separable fashion. However, images typically are statistically nonseparable, which motivates this investigation of nonseparable cubic convolution. This paper derives two new nonseparable, 2-D cubic-convolution kernels. The first kernel, with three parameters (designated 2D-3PCC), is the most general 2-D, piecewise-cubic interpolator defined on $[-2,2] \times[-2,2]$ with constraints for biaxial symmetry, diagonal (or $90^{\circ}$ rotational) symmetry, continuity, and smoothness. The second kernel, with five parameters (designated 2D-5PCC), relaxes the constraint of diagonal symmetry, based on the observation that many images have rotationally asymmetric statistical properties. This paper also develops a closed-form solution for determining the optimal parameter values for parametric cubic-convolution kernels with respect to ensembles of scenes characterized by autocorrelation (or power spectrum). This solution establishes a practical foundation for adaptive interpolation based on local autocorrelation estimates. Quantitative fidelity analyses and visual experiments indicate that these new methods can outperform several popular interpolation methods. An analysis of the error budgets for reconstruction error associated with blurring and aliasing illustrates that the methods improve interpolation fidelity for images with aliased components. For images with little or no aliasing, the methods yield results similar to other popular methods. Both 2D-3PCC and 2D-5PCC are low-order polynomials with small spatial support and so are easy to implement and efficient to apply.
\end{abstract}

Index Terms-Cubic convolution, image processing, interpolation, reconstruction, resampling.

\section{INTRODUCTION}

I MAGE interpolation is the process of defining a spatially continuous image from a set of discrete samples. Interpolation is fundamental to many digital image processing applications, particularly in operations requiring image resampling, such as scaling, registration, warping, and correction for geometric distortions. Interpolation commonly is implemented by convolving an image with a small kernel for the weighting function. Popular methods of interpolation by convolution include nearest neighbor interpolation, bi-linear interpolation, cubic B-spline interpolation, and piecewise-cubic convolution [1], [2].

Manuscript received December 6, 2004; revised August 15, 2005. This work was supported by the NSF Digital Government Program, Award EIA-0091530, and the NSF Science and Engineering Information Integration and Informatics Program, Award IIS-0431119. The associate editor coordinating the review of this manuscript and approving it for publication was Prof. Stanley J. Reeves.

The authors are with the Computer Science and Engineering Department, University of Nebraska, Lincoln NE 68588-0115 USA (e-mail: reich@cse.unl.edu).

Digital Object Identifier 10.1109/TIP.2006.873429
Cubic convolution has been used for image interpolation since the 1970s and provides a good compromise between computational complexity and interpolation accuracy [3]. Cubic convolution can be parameterized and then optimized either for general performance characteristics or for optimal fidelity over an image ensemble with specific characteristics [4], [5].

Traditionally, the cubic kernel has been derived in one dimension with one parameter and applied to two-dimensional (2-D) images in a separable fashion. However, images typically are statistically nonseparable. Reichenbach and Geng [6] derived a nonseparable, 2-D cubic kernel with two parameters (designated 2D-2PCC) and showed that it yielded better image interpolation accuracy than separable cubic convolution.

This paper relaxes constraints imposed on $2 \mathrm{D}-2 \mathrm{PCC}$ to develop two more-general, 2-D cubic-convolution kernels. The first kernel, with three parameters (designated 2D-3PCC), relaxes constraints on the kernel value at joints between pieces of the 2D-2PCC kernel [7]. The 2D-3PCC is the most general 2-D, piecewise-cubic interpolator defined on $[-2,2] \times[-2,2]$ with constraints for biaxial symmetry, diagonal symmetry (which, with biaxial symmetry, provides $90^{\circ}$ rotational symmetry), continuity, and smoothness. The second kernel, with five parameters (designated 2D-5PCC), relaxes the constraint of diagonal symmetry, based on the observation that many images have rotationally asymmetric statistical properties.

This paper also develops a closed-form solution for determining the optimal parameter values for 2D-3PCC and 2D-5PCC as a function of the scene autocorrelation (or power spectrum). The method for this closed-form solution also can be applied to variants of 2-D cubic convolution derived for other constraints. Experimental results, presented in the paper, illustrate two approaches for estimating the scene autocorrelation. The first approach uses parametric mathematical models to approximate the scene autocorrelation function. The second approach uses the observed autocorrelation function from a high-resolution image of a similar scene. For the first approach, the paper formulates a computationally efficient method based on a flexible, nonseparable scene model. This method can be implemented locally and when utilized with the closed-form solution for determining the optimal parameters supports fast, adaptive processing.

Quantitative fidelity analyses and visual experiments indicate that 2D-3PCC outperforms several popular interpolation methods for real scenes whose autocorrelation is relatively symmetric with $90^{\circ}$ rotation and that $2 \mathrm{D}-5 \mathrm{PCC}$ is a superior interpolator for scenes whose autocorrelation is rotationally asymmetric. In theoretical and experimental analyses, both 2D-3PCC and 2D-5PCC better balance error budget tradeoffs 
involving scene signal and aliasing and more closely resemble the unconstrained least-squares interpolator. In these analyses, 2D-5PCC is especially effective compared to separable methods for interpolating off-axes spatial frequencies. Both 2D-3PCC and 2D-5PCC are low-order polynomials with small spatial support and so are easy to implement and efficient to apply in the spatial domain.

The rest of this paper is organized as follows. Section II formulates the 2D-3PCC and 2D-5PCC interpolators subject to their constraints. Section III develops the closed-form solutions for determining the optimal parameters and illustrates parameterization based on Markov random field (MRF) scene models. Section IV analyzes quantitative performance and computational complexity. Section V demonstrates the visual quality, as well as the quantitative fidelity, for interpolation of example scenes. Section VI draws conclusions and describes ongoing and potential research on nonseparable cubic convolution.

\section{Formulations OF PARAMETRIC CUBiC CONVOLUTION}

This section outlines the derivation of equations that define 2D-3PCC and 2D-5PCC for image interpolation. Image interpolation attempts to recreate a continuous scene from discrete samples. Let $s(x, y),(x, y \in \mathbb{R})$, be a continuous $2-\mathrm{D}$ scene and $s[m, n],(m, n \in 0, \pm 1, \pm 2, \ldots)$, be an image consisting of uniformly spaced discrete samples from the scene (for notational convenience, the spatial coordinates are normalized in units of the sampling intervals without loss of generality). Interpolation of the original scene typically is implemented by convolving the image $s[m, n]$ with a continuous 2-D kernel $f(x, y),(x, y \in \mathbb{R})$

$$
r(x, y)=\sum_{m=-\infty}^{+\infty} \sum_{n=-\infty}^{+\infty} s[m, n] f(x-m, y-n)
$$

(in practice, the spatial extent of the image is finite, but that issue is not significant for the following derivations). In the Fourier frequency domain

$$
\hat{r}(u, v)=\hat{f}(u, v) \sum_{\mu=-\infty}^{+\infty} \sum_{\nu=-\infty}^{+\infty} \hat{s}(u-\mu, v-\nu)
$$

where $\hat{r}(u, v), \hat{f}(u, v)$, and $\hat{s}(u, v)$ are the Fourier transforms of the interpolated result $r(x, y)$, interpolation kernel $f(x, y)$, and the scene $s(x, y)$ respectively. Many popular image interpolation methods are defined in this way, including nearest-neighbor interpolation, bi-linear interpolation, cubic-spline interpolation, and cubic convolution [1], [2], [8].

The piecewise cubic-polynomial kernel over the region $[-2,2] \times[-2,2]$ has 16 unit-sized pieces, each piece with 16 parameters, e.g.,

$$
f(x, y)=\sum_{j=0}^{3} \sum_{k=0}^{3} a_{j k} x^{j} y^{k}, \quad 0 \leq x \leq 1,0 \leq y \leq 1
$$

which allows $16 \times 16=256$ degrees of freedom or parameters (the parameter subscripts indicate the powers for each spatial variable). The large number of parameters can be reduced by constraining the kernel. In Section II-A, 2D-3PCC is formulated subject to traditional constraints for image interpolation: symmetry, continuity, and smoothness [5], [9], [10]. Section II-B relaxes the constraint of $90^{\circ}$ rotational symmetry to generate 2D-5PCC. The traditional interpolation constraints for continuity, smoothness, and symmetry yield 2D-3PCC and 2D-5PCC, but cubic convolution could be formulated in a similar manner for other constraints, e.g., for special scene characteristics, for variable constraints for adaptive filtering [11], or for different numbers of parameters.

\section{A. Formulation of $2 D-3 P C C$}

$2 \mathrm{D}-3 \mathrm{PCC}$ is the most general 2-D piecewise-cubic kernel over the region $[-2,2] \times[-2,2]$ with constraints for flat-field interpolation, biaxial and diagonal symmetry, continuity, and smoothness. This formulation eliminates constraints on 2D-2PCC [6]

$$
\forall(x, y), f(x, 1)=f(1, y) \equiv 0
$$

which is not required for interpolation. With these constraints, the 2-D cubic-convolution kernel can be reduced to a function of only three coefficients $\left(a_{33}, a_{32}, a_{30}\right)$ [7]. The four pieces in the first quadrant (solved using symbolic mathematics software, e.g., Maple or Mathematica) are shown in (5), at the bottom of the page, with the pieces in other quadrants defined by biaxial symmetry: $f(-x, y)=f(x, y)$ and $f(x,-y)=f(x, y)$. 2D-3PCC also can be formulated in terms of the one parameter

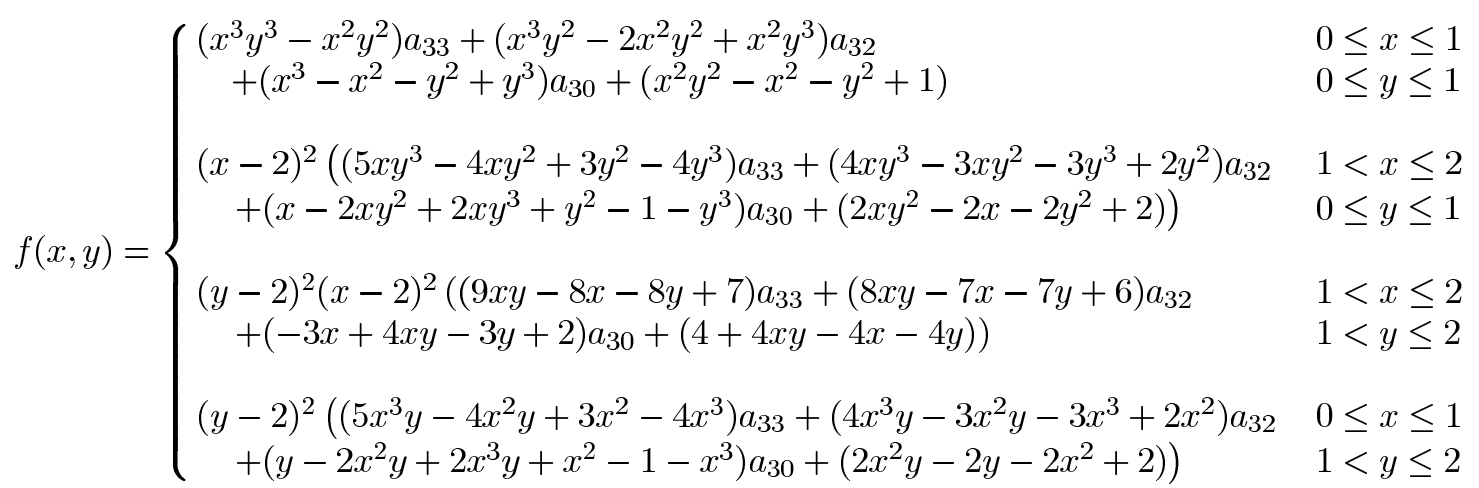




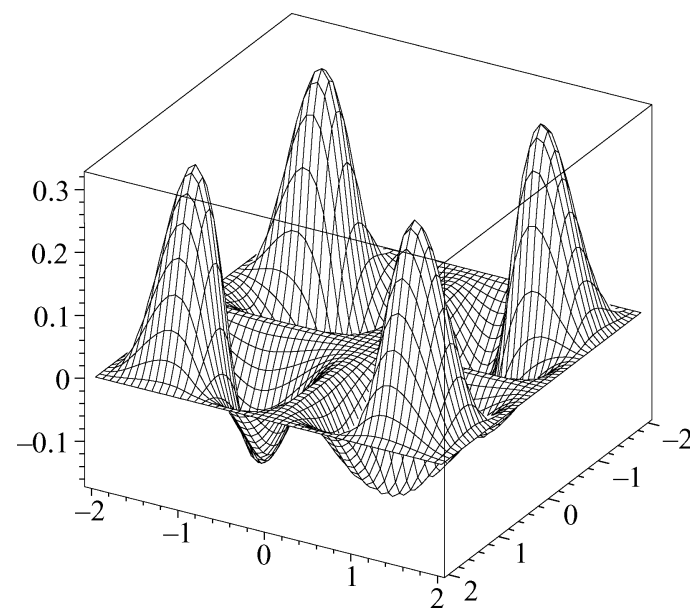

Fig. 1. The 2D-3PCC component $f_{2}$ in (8).

$\alpha$ of the traditional separable filter [4], [5], the second parameter $\beta$ derived in [6], and an additional parameter $\gamma$ with the following substitutions:

$$
\begin{aligned}
& a_{30}=\alpha+2 \\
& a_{33}=\beta+(\alpha+2)^{2} \\
& a_{32}=\gamma-(\alpha+2)(\alpha+3)-\beta .
\end{aligned}
$$

With these parameters, 2D-3PCC can be rewritten as

$$
\begin{aligned}
f(x, y)=\left(f_{0}(x)+\alpha f_{1}(x)\right) & \left(f_{0}(y)+\alpha f_{1}(y)\right) \\
& +\beta f_{1}(x) f_{1}(y)+\gamma f_{2}(x, y)
\end{aligned}
$$

where $f_{0}$ and $f_{1}$ are the two component functions of 2D-2PCC [6] and $f_{2}$ is an additional nonseparable function parameterized by $\gamma$, shown in the equation at the bottom of the page. The $f_{2}$ term, pictured in Fig. 1, is well suited to interpolate nonseparable, diagonal features.

\section{B. Formulation of $2 D-5 P C C$}

The 2D-5PCC is derived with constraints for biaxial symmetry, continuity, smoothness, and flat-field interpolation. It is, however, not subject to the constraint of diagonal (or $90^{\circ}$ rotational) symmetry. By removing the constraint of diagonal symmetry, cubic convolution can be tailored for images that have more detail along one axis than the other. With these constraints, the nonseparable, biaxial symmetric, 2D-5PCC kernel can be reduced to

$$
\begin{aligned}
f(x, y)=a_{33} f_{33}(x, y)+a_{32} f_{32}(x, y)+a_{23} f_{23}(x, y) \\
+a_{30} f_{30}(x, y)+a_{03} f_{03}(x, y)+f_{00}(x, y)
\end{aligned}
$$

where $\left(a_{33}, a_{32}, a_{23}, a_{30}, a_{03}\right)$ are the five parameters and the functions $\left(f_{33}, f_{32}, f_{23}, f_{30}, f_{03}, f_{00}\right)$ are defined in Fig. 2. The component functions $f_{33}$ and $f_{00}$ are the same as their counterparts in 2D-3PCC [defined in (5)]; $f_{32}$ and $f_{23}$ are split from the component for parameter $a_{32}$ in 2D-3PCC; and $f_{30}$ and $f_{03}$ are split from the component for parameter $a_{30}$ in 2D-3PCC. Fig. 3 shows the spatial-domain point spread functions of 2D-5PCC components. Components $f_{30}(x, y)$ and $f_{32}(x, y)$ are well suited to enhance details along the $x$ axis and $f_{03}(x, y)$ and $f_{23}(x, y)$ are well suited to enhance details along the $y$ axis.

\section{PARAMETERIZATION}

\section{A. Closed-Form Solutions for Optimal Parameter Values}

This section develops closed-form solutions for the optimal parameters of 2D-3PCC and 2D-5PCC, based on an analysis of the mean square error (MSE). By Rayleigh's theorem [12], the MSE $\epsilon^{2}$ for interpolation can be analyzed in either the spatial or frequency domain

$$
\begin{aligned}
\epsilon^{2} & =\iint|r(x, y)-s(x, y)|^{2} d x d y \\
& =\iint|\hat{r}(u, v)-\hat{s}(u, v)|^{2} d u d v .
\end{aligned}
$$

The optimal parameters minimize expected MSE $\bar{\epsilon}^{2}=E\left\{\epsilon^{2}\right\}$ for an ensemble of scenes, given the autocorrelation (or power spectrum). If co-aliased components of the sampled scene are uncorrelated [13], the expected MSE can be expressed in terms of the scene power spectrum and the interpolation transfer function

$$
\begin{aligned}
\bar{\epsilon}^{2}=\iint\left(\hat{\Phi}_{s}(u, v)-2 \hat{f}(u, v) \hat{\Phi}_{s}(u, v)\right. & \\
& \left.+|\hat{f}(u, v)|^{2} \hat{\Phi}_{p}(u, v)\right) d u d v
\end{aligned}
$$

where $\hat{\Phi}_{s}(u, v)=E\left\{|\hat{s}(u, v)|^{2}\right\}$ is the scene power spectrum and

$$
\hat{\Phi}_{p}(u, v)=\sum_{\mu} \sum_{\nu} \hat{\Phi}_{s}(u-\mu, v-\nu)
$$

is the image power spectrum (with aliasing of the scene components). For convenience and without loss of generality, scenes are normalized so that the mean and variance are zero and one respectively.

For 2D-5PCC, the expected MSE $\bar{\epsilon}^{2}$ is a quadratic function of the parameters, $\left(a_{33}, a_{32}, a_{23}, a_{30,03}\right)$, so the optimal parameter

$$
f_{2}(x, y)= \begin{cases}(x+y-2) x^{2} y^{2} & 0 \leq x \leq 1,0 \leq y \leq 1 \\ (4 x y-3 x-3 y+2) y^{2}(x-2)^{2} & 1<x \leq 2,0 \leq y \leq 1 \\ (8 x y-7 x-7 y+6)(x-2)^{2}(y-2)^{2} & 1<x \leq 2,1<y \leq 2 \\ (4 x y-3 x-3 y+2) x^{2}(y-2)^{2} & 0 \leq x \leq 1,1<y \leq 2\end{cases}
$$




$$
\begin{aligned}
& f_{33}(x, y)= \begin{cases}x^{3} y^{3}-x^{2} y^{2} & 0 \leq x \leq 1,0 \leq y \leq 1 \\
(x-2)^{2}\left(5 x y^{3}-4 x y^{2}-4 y^{3}+3 y^{2}\right) & 1<x \leq 2,0 \leq y \leq 1 \\
(x-2)^{2}(y-2)^{2}(9 x y-8 x-8 y+7) & 1<x \leq 2,1<y \leq 2 \\
(y-2)^{2}\left(5 x^{3} y-4 x^{2} y-4 x^{3}+3 x^{2}\right) & 0 \leq x \leq 1,1<y \leq 2\end{cases} \\
& f_{32}(x, y)= \begin{cases}x^{3} y^{2}-x^{2} y^{2} & 0 \leq x \leq 1,0 \leq y \leq 1 \\
(x-2)^{2}\left(x y^{2}-y^{2}\right) & 1<x \leq 2,0 \leq y \leq 1 \\
(y-2)^{2}(x-2)^{2}(4 x y-4 y-3 x+3) & 1<x \leq 2,1<y \leq 2 \\
(y-2)^{2}\left(4 x^{3} y-4 x^{2} y-3 x^{3}+3 x^{2}\right) & 0 \leq x \leq 1,1<y \leq 2\end{cases} \\
& f_{23}(x, y)= \begin{cases}x^{2} y^{3}-x^{2} y^{2} & 0 \leq x \leq 1,0 \leq y \leq 1 \\
(x-2)^{2}\left(4 x y^{3}-4 x y^{2}-3 y^{3}+3 y^{2}\right) & 1<x \leq 2,0 \leq y \leq 1 \\
(y-2)^{2}(x-2)^{2}(4 x y-4 x-3 y+3) & 1<x \leq 2,1<y \leq 2 \\
(y-2)^{2}\left(x^{2} y-x^{2}\right) & 0 \leq x \leq 1,1<y \leq 2\end{cases} \\
& f_{30}(x, y)= \begin{cases}x^{3}-x^{2} & 0 \leq x \leq 1,0 \leq y \leq 1 \\
(x-2)^{2}(x-1) & 1<x \leq 2,0 \leq y \leq 1 \\
(x-2)^{2}(y-2)^{2}(2 x y-2 y-x+1) & 1<x \leq 2,1<y \leq 2 \\
(y-2)^{2}\left(2 x^{3} y-2 x^{2} y-x^{3}+x^{2}\right) & 0 \leq x \leq 1,1<y \leq 2\end{cases} \\
& f_{03}(x, y)= \begin{cases}y^{3}-y^{2} & 0 \leq x \leq 1,0 \leq y \leq 1 \\
(x-2)^{2}\left(2 x y^{3}-2 x y^{2}-y^{3}+y^{2}\right) & 1<x \leq 2,0 \leq y \leq 1 \\
(y-2)^{2}(x-2)^{2}(2 x y-2 x-y+1) & 1<x \leq 2,1<y \leq 2 \\
(y-2)^{2}(y-1) & 0 \leq x \leq 1,1<y \leq 2\end{cases} \\
& f_{00}(x, y)= \begin{cases}x^{2} y^{2}-x^{2}-y^{2}+1 & 0 \leq x \leq 1,0 \leq y \leq 1 \\
(x-2)^{2}\left(2 x y^{2}-2 y^{2}-2 x+2\right) & 1<x \leq 2,0 \leq y \leq 1 \\
(y-2)^{2}(x-2)^{2}(4 x y-4 y-4 x+4) & 1<x \leq 2,1<y \leq 2 \\
(y-2)^{2}\left(2 x^{2} y-2 x^{2}-2 y+2\right) & 0 \leq x \leq 1,1<y \leq 2 .\end{cases}
\end{aligned}
$$

Fig. 2. Component equations for $2 \mathrm{D}-5 \mathrm{PCC}$ in (8).

values can be derived by substituting the Fourier transform of the kernel from (8) into (10), computing the partial derivatives of the expected MSE $\bar{\epsilon}^{2}$ with respect to the parameters, and solving for simultaneous equality with zero

$$
\frac{\partial \bar{\epsilon}^{2}}{\partial a_{33}}=\frac{\partial \bar{\epsilon}^{2}}{\partial a_{32}}=\frac{\partial \bar{\epsilon}^{2}}{\partial a_{23}}=\frac{\partial \bar{\epsilon}^{2}}{\partial a_{30}}=\frac{\partial \bar{\epsilon}^{2}}{\partial a_{03}}=0 .
$$

This yields five equations for the optimal parameter values

$$
\begin{aligned}
& \iint \hat{g}_{i}(u, v)\left(\hat{f}(u, v)-\hat{f}_{00}(u, v)\right) \hat{\Phi}_{p}(u, v) d u d v \\
& =\iint \hat{g}_{i}(u, v)\left(\hat{\Phi}_{s}(u, v)-\hat{f}_{00}(u, v) \hat{\Phi}_{p}(u, v)\right) d u d v
\end{aligned}
$$

for $\hat{g}_{0}=\hat{f}_{33}, \hat{g}_{1}=\hat{f}_{32}, \hat{g}_{2}=\hat{f}_{23}, \hat{g}_{3}=\hat{f}_{30}$, and $\hat{g}_{4}=\hat{f}_{03}$.

With matrix notation, (13) can be written as $H A=K$

$$
\left[\begin{array}{lllll}
h_{00} & h_{01} & h_{02} & h_{03} & h_{04} \\
h_{10} & h_{11} & h_{12} & h_{13} & h_{14} \\
h_{20} & h_{21} & h_{22} & h_{23} & h_{24} \\
h_{30} & h_{31} & h_{32} & h_{33} & h_{34} \\
h_{40} & h_{41} & h_{42} & h_{43} & h_{44}
\end{array}\right]\left[\begin{array}{l}
a_{33} \\
a_{32} \\
a_{23} \\
a_{30} \\
a_{03}
\end{array}\right]=\left[\begin{array}{l}
k_{0} \\
k_{1} \\
k_{2} \\
k_{3} \\
k_{4}
\end{array}\right]
$$

where

$$
\begin{aligned}
& h_{i j}=\iint \hat{g}_{i}(u, v) \hat{g}_{j}(u, v) \hat{\Phi}_{p}(u, v) d u d v \\
& =\sum_{m=-3}^{3} \sum_{n=-3}^{3}\left(g_{i} \otimes g_{j}\right)[m, n] \Phi_{p}[m, n] \\
& k_{i}=\iint \hat{g}_{i}(u, v)\left(\hat{\Phi}_{s}(u, v)-\hat{f}_{00}(u, v) \hat{\Phi}_{p}(u, v)\right) d u d v \\
& =\int_{-2}^{2} \int_{-2}^{2} g_{i}(x, y) \Phi_{s}(x, y) d x d y \\
& -\sum_{m=-3}^{3} \sum_{n=-3}^{3}\left(g_{i} \otimes f_{00}\right)[m, n] \Phi_{p}[m, n]
\end{aligned}
$$

where " $\otimes$ " denotes convolution and $\Phi_{p}$ is the image autocorrelation

$$
\Phi_{p}[m, n]=\iint \Phi_{s}(x, y) \delta(x-m, y-n) d x d y
$$

where $\delta(x)$ is the impulse function. The equivalences of both (15) and (16) are guaranteed by the Plancherel-Parseval identity [14]. Then, the optimal parameter values can be solved ef- 


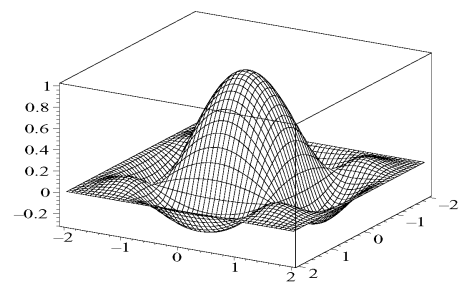

(a)

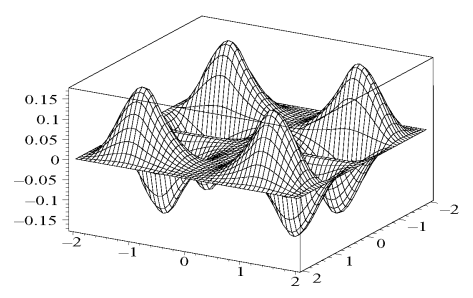

(d)

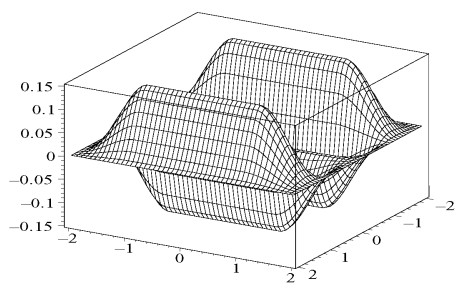

(b)

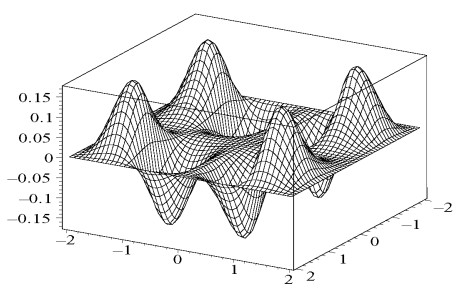

(e)

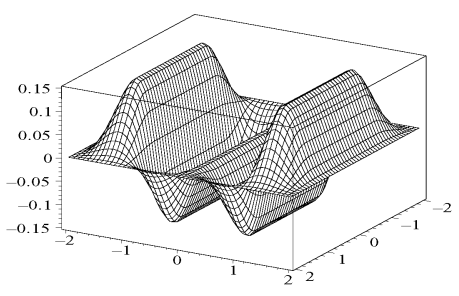

(c)

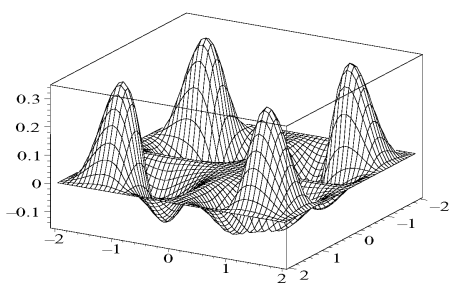

(f)

Fig. 3. The 2D-5PCC components in the spatial domain. (a) $f_{00}(x, y)$; (b) $f_{30}(x, y)$; (c) $f_{03}(x, y)$; (d) $f_{32}(x, y)$; (e) $f_{23}(x, y)$; and (f) $f_{33}(x, y)$.

ficiently in either the spatial domain or the Fourier frequency domain as

$$
A=H^{-1} K
$$

if the symmetric matrix $H$ is well-conditioned ( $H$ is well-conditioned except as the power spectrum approaches an impulse function, in which case any unitary, flat-field interpolator works equally well).

Computing the optimal parameter values requires the scene autocorrelation $\Phi_{s}$ only in the region $[-2,2] \times[-2,2]$ (because the cubic convolution kernel is zero outside that support) and the spatial support of the image autocorrelation $\Phi_{p}$ only on the grid points in the region $[-3,3] \times[-3,3]$ [because $\left(g_{i} \otimes g_{j}\right)[m, n]$ and $\left(g_{i} \otimes f_{00}\right)[m, n]=0$ are zero outside that support]. This dependency on local autocorrelation facilitates the development of adaptive processing.

Using the same approach, the closed-form solution for the optimal parameters for 2D-3PCC is

$$
\left[\begin{array}{l}
a_{33} \\
a_{32} \\
a_{30}
\end{array}\right]=\left[\begin{array}{lll}
h_{00}^{\prime} & h_{01}^{\prime} & h_{02}^{\prime} \\
h_{10}^{\prime} & h_{11}^{\prime} & h_{12}^{\prime} \\
h_{20}^{\prime} & h_{21}^{\prime} & h_{22}^{\prime}
\end{array}\right]^{-1}\left[\begin{array}{c}
k_{0} \\
k_{1}+k_{2} \\
k_{3}+k_{4}
\end{array}\right]
$$

where

$$
\begin{aligned}
h_{i j}^{\prime} & =\iint \hat{g}_{i}^{\prime}(u, v) \hat{g}_{j}^{\prime}(u, v) \hat{\Phi}_{p}(u, v) d u d v \\
& =\sum_{m=-3}^{3} \sum_{n=-3}^{3}\left(g_{i}^{\prime} \otimes g_{j}^{\prime}\right)[m, n] \Phi_{p}[m, n]
\end{aligned}
$$

for

$$
\begin{aligned}
& \hat{g}_{0}^{\prime}=\hat{g}_{0}=\hat{f}_{33} \\
& \hat{g}_{1}^{\prime}=\hat{g}_{1}+\hat{g}_{2}=\hat{f}_{32}+\hat{f}_{23} \\
& \hat{g}_{2}^{\prime}=\hat{g}_{3}+\hat{g}_{4}=\hat{f}_{30}+\hat{f}_{03} .
\end{aligned}
$$

If desired, the optimal values of parameters $\alpha, \beta$, and $\gamma$ can be derived by substitution. From (6)

$$
\begin{aligned}
& \alpha=a_{30}-2 \\
& \beta=a_{33}-a_{30}^{2} \\
& \gamma=a_{33}+a_{32}+a_{30} .
\end{aligned}
$$

The approach described in this section yields a closed-form solution for the optimal parameters for 2-D cubic convolution subject to two different sets of constraints-one set of constraints for 2D-3PCC and one set of constraints for 2D-5PCC. The same approach can be used to derive the optimal parameters for 2-D cubic convolution formulated for other constraints.

\section{B. Parameterization Examples for Markov Random Field Models}

As described in Section III-A, the optimal parameter values depend upon the scene autocorrelation function. One approach for approximating the scene autocorrelation function is to use a mathematical model. Another approach is to use the actual autocorrelation function from one or more high-resolution images of a similar scene(s). The MRF scene model is used widely in image and vision computing [15] and has a nonseparable autocorrelation function (and power spectrum). Appendix A describes two autocorrelation models based on MRF scenes. One model, for an isotropic MRF, is a radially symmetric autocorrelation function with one parameter: mean spatial detail (MSD) $d$, which can be interpreted as the average distance between details in the scene (not the image) along a line in any direction, measured in pixel units (the interval between samples in the image). A scene with larger spatial features has a larger MSD. The second autocorrelation model, based an isotropic MRF subject to affine transformation (without translation), is not radially symmetric and so allows directionally dependent levels of detail. The analyses of this section consider MRF scenes in a $512 \times 512$ array down-sampled to $128 \times 128$ pixels which are to be interpolated back to $512 \times 512$. The optimal parameters are computed from power spectra (or autocorrelation functions) in $512 \times 512$ arrays with frequencies to \pm 2 cycles/pixel. 


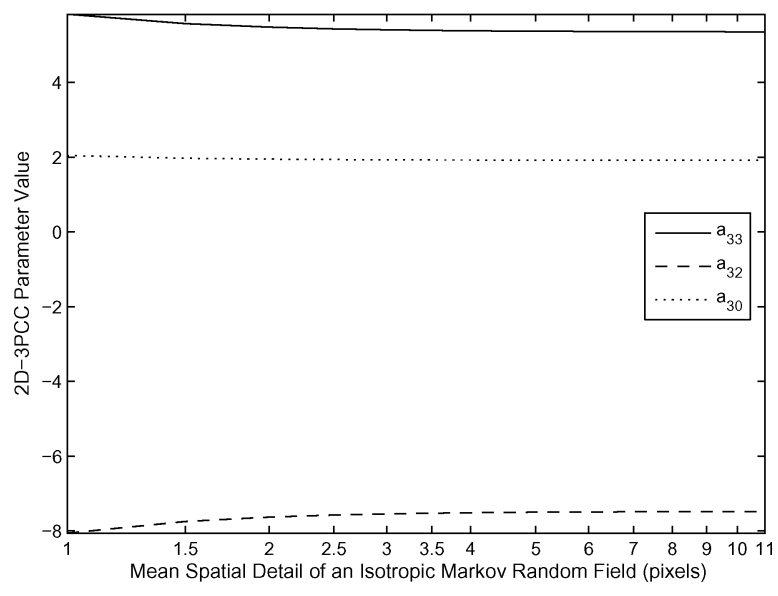

(a)

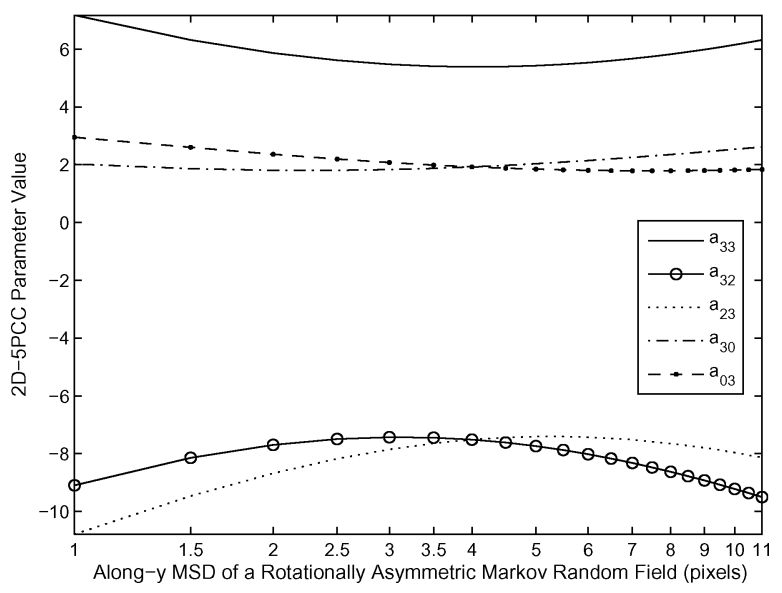

(b)

Fig. 4. Optimal parameter values for: (a) 2D-3PCC for an isotropic MRF as a function of MSD $d$ and (b) 2D-5PCC for a rotationally asymmetric MRF as a function of MSD along-y $d_{y}$ (with MSD along- $x d_{x}=4$ pixels and no rotation $d_{c}=0$ ).

Based on the isotropic MRF scene model with the autocorrelation formulated by (27), Fig. 4(a) graphs the optimal parameter values for 2D-3PCC as a function of the MSD $d$ (because the autocorrelation model is rotationally symmetric $2 \mathrm{D}-5 \mathrm{PCC}$ is identical to 2D-3PCC). For $d=1$ pixel, the optimal $a_{33}, a_{32}$, and $a_{30}$ are 5.83, -8.06 , and 2.04, respectively. As the MSD $d$ increases, both $a_{33}$ and $a_{30}$ decrease slightly and $a_{32}$ increases slightly. For MSD $d=10$ pixels, the optimal values are 5.35, -7.48 , and 1.92. For the isotropic MRF scene model, the values of the 2D-3PCC parameters are relatively independent of the MSD of the scene, especially for $d>=4$ pixels. This means that 2D-3PCC is robust for a mismatch between the MSD presumed in parameterizing the kernel and the MSD of the actual scene.

Fig. 4(b) graphs the optimal parameter values for 2D-5PCC, based on the rotationally asymmetric autocorrelation model in (28), with $d_{x}=4$ (i.e., MSD along- $x$ is four pixels), $d_{c}=0$ (i.e., no rotation), and the optimal parameter values shown as functions of the MSD along- $y d_{y}$. For $d_{y}=d_{x}$ at four pixels, $2 \mathrm{D}-5 \mathrm{PCC}$ is identical to 2D-3PCC, so the pairs of parameters are equal, $a_{23}=a_{32}$ and $a_{30}=a_{03}$. Also, parameter $a_{33}$ reaches its minimum value where $d_{y}=d_{x}$. For $d_{y}<d_{x}, a_{23}<a_{32}$ and $a_{30}<a_{03}$. In this range, as the MSD along- $y$ increases, the differences between the two pairs of parameters narrow. For $d_{y}>d_{x}, a_{23}>a_{32}$ and $a_{30}>a_{03}$. In this range, as the MSD along- $y$ increases, the differences between the two pairs of parameters increase. This result can be understood by observing that $a_{32}$ and $a_{23}$ (as well as $a_{30}$ and $a_{03}$ ) emphasize details along different directions. Because the parameter values change relatively slowly with directional MSD, 2D-5PCC is relatively robust for a small mismatch between the directional MSD presumed in parameterizing the kernel and the directional MSD of the actual scene.

\section{Performance Analyses}

\section{A. MSE Performance}

This section analyzes interpolation quality measured by the fidelity [16] of the interpolated image $r(x, y)$ [in (1)] to the scene $s(x, y)$. Fidelity is a normalized measure based on the MSE

$$
\mathcal{F}=1-\frac{\epsilon^{2}}{\iint \hat{\Phi}_{s}(u, v) d u d v} .
$$

The greatest fidelity possible is 1.0 , when the MSE is 0 . In this section, the expected MSE is computed from (10) based on MRF scenes in a $512 \times 512$ array, down-sampled to $128 \times 128$ pixel images which are interpolated back to $512 \times 512$ and compared to the original scene.

Fig. 5(a) graphs fidelity as a function of the MSD $d$ for the isotropic MRF scene model [with the autocorrelation formulated in (27)] (for this scene model, 2D-5PCC is identical to 2D-3PCC). Fidelity is shown for interpolation by the unconstrained least-squares interpolator, 2D-3PCC, 2D-2PCC, separable bicubic convolution with $\alpha=-0.5$, cubic B-spline interpolation [1], [17], and cubic (o-Moms) interpolation [10], [18]. As expected, the unconstrained least-squares interpolator (a Wiener or kriging filter without constraints on support or resolution) has the best fidelity, but this filter has unconstrained spatial support and unconstrained frequency resolution and so requires computationally expensive Fourier-domain processing. Of the constrained interpolation methods, 2D-3PCC has the best fidelity across the wide range of scales for the isotropic MRF scene model. For images with aliasing, 2D-3PCC performs especially well compared to the other constrained interpolation methods. For example, with MSD equal to four pixels, 2D-3PCC has fidelity of 0.902, compared to 0.890 for cubic o-Moms interpolation and 0.895 for cubic B-spline interpolation. For images with little high-frequency content, and so with little aliasing, all of the interpolation methods perform well and yield similar fidelity. For example, with MSD equal to 31 pixels [nearly one-quarter of the image size and beyond the range of Fig. 5(a)], all of the interpolation methods have fidelity of 0.993 .

Fig. 5(b) graphs the fidelity as a function of the MSD along- $y$ $d_{y}$ for the rotationally asymmetric MRF scene model with the autocorrelation formulated in (28), $d_{x}=4$ (i.e., MSD along $x$ is four pixels), and $d_{c}=0$. Fidelity is shown only for interpolation by the unconstrained least-squares interpolator, 2D-5PCC, 


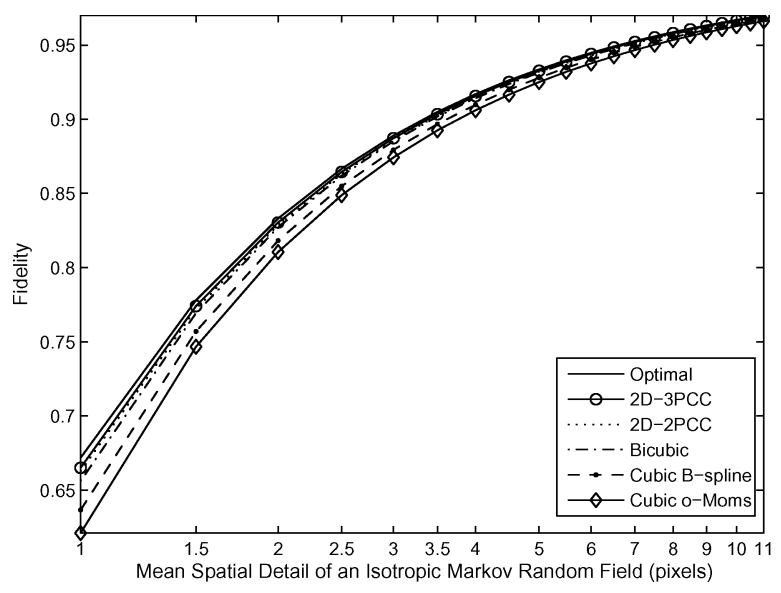

(a)

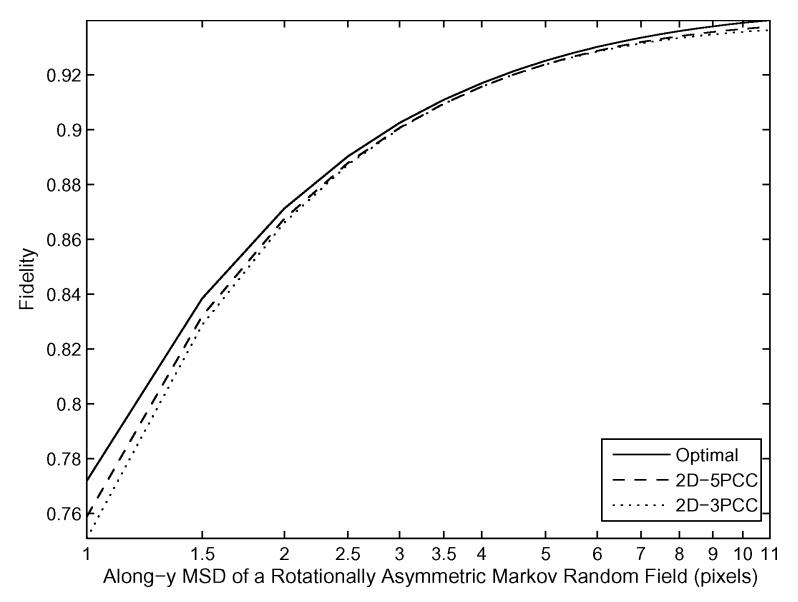

(b)

Fig. 5. Interpolation fidelity for: (a) 2D-3PCC for an isotropic MRF as a function of MSD $d$ and (b) 2D-5PCC for a rotationally asymmetric MRF as a function of MSD along- $y d_{y}$ (with MSD along- $x d_{x}=4$ pixels and no rotation $d_{c}=0$ ).

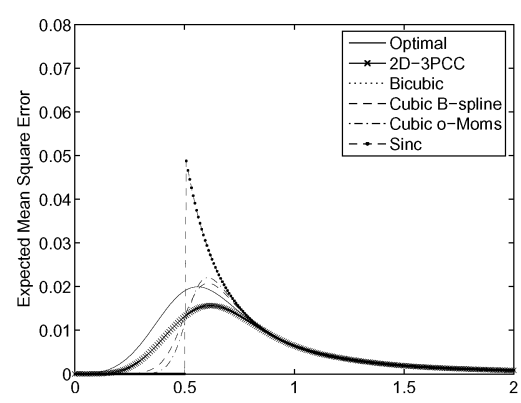

(a)

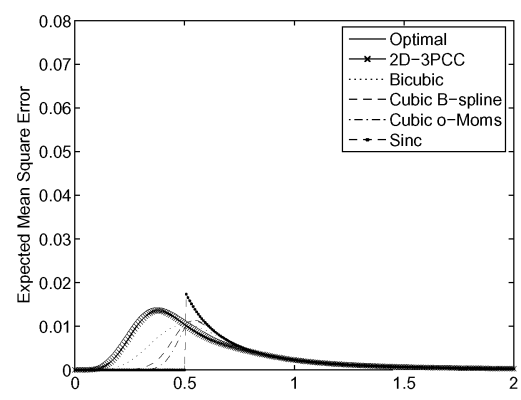

(d)

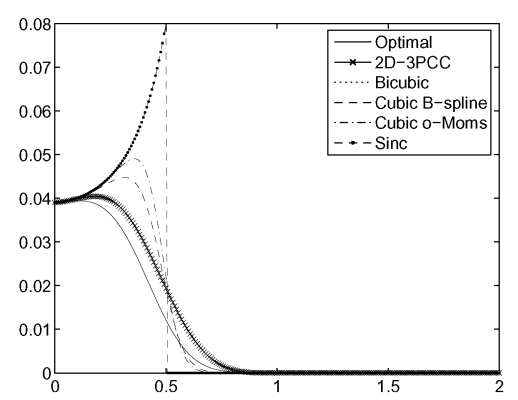

(b)

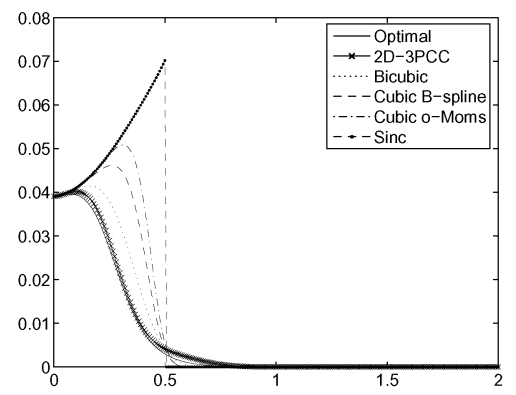

(e)

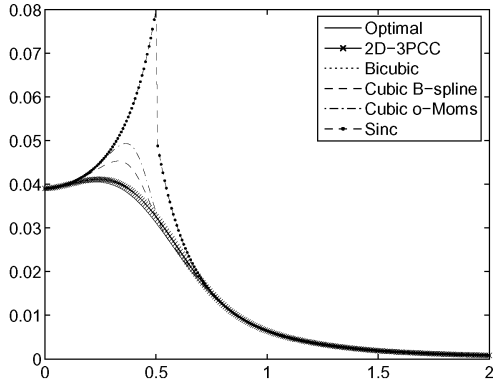

(c)

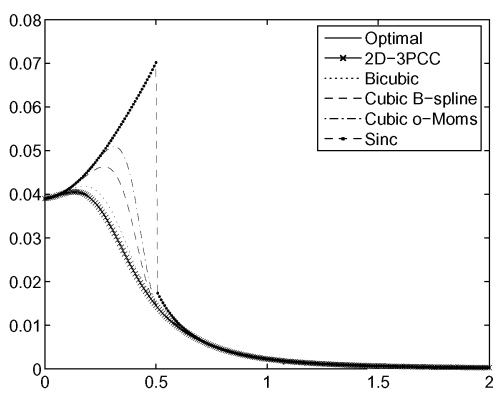

(f)

Fig. 6. Two-dimensional slices of the error budget components $e_{s}(u, v)$ and $e_{a}(u, v)$ and the total error budget for the isotropic MRF scene model with MSD $d=$ 4 pixels. The top row is for horizontal slices with $v=0$ and the bottom row is for diagonal slices with $u=v .\left(\right.$ a) $e_{s}(u, 0) ;(\mathrm{b}) e_{a}(u, 0) ;(\mathrm{c}) e_{s}(u, 0)+e_{a}(u, 0)$; (d) $e_{s}(u, u) ;(\mathrm{e}) e_{a}(u, u)$; and (f) $e_{s}(u, u)+e_{a}(u, u)$.

and 2D-3PCC, to illustrate the value of the additional parameters for scenes with rotationally asymmetric autocorrelation (or power spectrum). As the MSD along- $y d_{y}$ diverges from the MSD along- $x$, the superiority of 2D-5PCC over 2D-3PCC becomes more significant.

The superior performance of 2D-3PCC and 2D-5PCC can be analyzed by evaluating the expected mean square error (MSE) in (10) as an error budget equation [19]

$$
\bar{\epsilon}^{2}=\iint\left(e_{s}(u, v)+e_{a}(u, v)\right) d u d v
$$

where

$$
e_{s}(u, v)=|1-\hat{f}(u, v)|^{2} \hat{\Phi}_{s}(u, v)
$$

accounts for the error caused by alteration of the scene signal components at frequencies where $\hat{f}(u, v)$ is not equal to 1 and

$$
e_{a}(u, v)=|\hat{f}(u, v)|^{2} \sum_{(\mu, \nu) \neq(0,0)} \hat{\Phi}_{s}(u-\mu, v-\nu)
$$

accounts for the error caused by interpolation of the aliased components. The expected mean square error (MSE) $\bar{\epsilon}^{2}$ of interpolation is determined by not only the interpolator $\hat{f}(u, v)$, but also the scene power spectrum $\hat{\Phi}_{s}(u, v)$.

Fig. 6 plots slices of the error budget components $e_{s}(u, v)$ and $e_{a}(u, v)$ as well as the total error budget for the isotropic MRF scene model with MSD $d=4$ pixels. In the baseband $(|u|,|v| \leq 0.5$, the Nyquist limit), cubic B-spline and cubic o-Moms interpolation have flatter frequency responses than cubic convolution, and so have smaller signal error $e_{s}(u, v)$ but 
larger aliasing error $e_{a}(u, v)$. The sinc function causes no blurring in the baseband and so has no signal error, but does nothing to attenuate aliased components and so has large aliasing error. At frequencies beyond the Nyquist limit, cubic convolution has smaller signal error $e_{s}(u, v)$, but larger aliasing error $e_{a}(u, v)$. Beyond the Nyquist limit, the sinc function has zero aliasing error, but removes all signal. The total error budget beyond the Nyquist frequency is nearly the same for all methods except the sinc, but in the baseband cubic convolution has the smallest total error of all of the constrained methods and performs nearly as well as the unconstrained least-squares interpolator across the spectrum. The error budget for 2D-3PCC is nearly the same as for separable bicubic convolution at along-axes frequencies [Fig. 6(a)-(c)], but 2D-3PCC outperforms separable bicubic convolution at off-axes frequencies [Fig. 6(d)-(f)].

The optimal parameters for 2D-3PCC balance the error budget tradeoff between signal error and aliasing error. In this example, cubic convolution performs better than cubic B-spline and cubic o-Moms interpolation because cubic convolution deals better with aliasing. Most images have aliased components (including all images with edges), so accounting for aliasing in interpolation is important. Thevenaz et al. [10, pp. 748-749] noted: "[T]he class of band-limited functions represents but a tiny fraction of all possible functions; moreover, they often give a distorted view of the physical reality in an imaging context...Further, there exist obviously no way at all to perform any kind of antialiasing filter on physical matter (before sampling)." For example, it would be undesirable to prefilter elevation data obtained on a 2-D grid before interpolation, even if aliasing is present. As MSD increases, aliasing of frequency components beyond the Nyquist limit becomes less significant, and, as illustrated in Fig. 5(a), interpolation can be performed with greater fidelity and all of the interpolators have similar performance.

\section{B. Computational Complexity}

The computational cost of parametric cubic convolution consists of parameter optimization and nonseparable convolution. Parameter optimization is required only once for an ensemble or statistical class of images (or subimages for adaptive processing) and so typically can be performed off-line in time-sensitive applications. Furthermore, as seen in Fig. 4, 2-D cubic convolution is robust with respect to the autocorrelation function, so the same parameters can be used over a range of fairly similar images. Computing an autocorrelation function typically is performed using Fourier methods with computational complexity $O\{N \log N\}$, where $N$ is the number of pixels. The approach described in Appendix B for fitting a mathematical model to the image autocorrelation function requires evaluation of the three $7 \times 7$ matrices in (31). Given the scene autocorrelation function, computing the optimal parameters in (18) requires the computation and inversion of a $3 \times 3$ array for $2 \mathrm{D}-3 \mathrm{PCC}$ or a $5 \times 5$ array for $2 \mathrm{D}-5 \mathrm{PCC}$.

Cubic convolution requires determination of the weights for each pixel and application of the weights to the pixel values. For some interpolation tasks, such as zooming by an integer factor, the kernel weights can be precomputed. The nonseparable 2-D cubic kernel can be computed for 16 pixel locations in the $[-2,2] \times[-2,2]$ support with 204 floating-point multiplies and adds (MADDs). For $2 \times 2$ zoom, three such sets of kernel values are required (the fourth set, at the sample points, is the identity kernel). Then, for each output value, applying the kernel to the 16 pixels in the support requires 16 MADDs, which is the minimum computation required for any kernel with support that encompasses 16 pixels. For some other interpolation tasks, it may be necessary to compute the kernel weights for each output value. In such cases, evaluating and applying a nonseparable cubic (220 MADDs) is more costly than evaluating and applying a separable bicubic convolution (which requires 44 MADDs) or cubic B-spline interpolation (which also requires 44 MADDs if the computation for prefiltering is discounted [20]). It is possible to precompute the kernel on a dense grid and use table lookup to obtain the weights [21], but there is a loss in interpolation fidelity. Two-dimensional cubic convolution is amenable to parallelization, using either data partitioning or pipelining.

\section{EXPERIMENTAL RESULTS}

The MRF scene model facilitates quantitative performance assessment of interpolation methods. For many image applications, another important performance measure is the visual quality produced by interpolation. Using examples of real images, this section considers visual quality, as well as quantitative fidelity. Several digital images are used as scenes, down-sampled at varying densities, interpolated back to the original size, and compared to the original scenes. The experiments compare 2D-3PCC and 2D-5PCC with 2D-2PCC, the unconstrained least-squares interpolator, separable bicubic convolution, cubic B-spline interpolation, and edge-directed interpolation [22]. The actual scene power spectra are used to derive the unconstrained least-squares interpolators (which are applied in the frequency domain) in order to benchmark the optimal fidelity. The autocorrelations for optimizing the cubic convolution kernels are estimated (as typically is required in practice) based on either the MRF scene model (fit to the image autocorrelation as described in Appendix B) or on similar "training" images. Separable bicubic convolution and cubic B-spline interpolation are implemented by MATLAB's "INTERP2" function. The program for edge-directed interpolation was downloaded from Prof. Li's website [23].

\section{A. Scene Autocorrelation From MRF Model}

Fig. 7(a) shows an image of the moon from the USC-SIPI database [24]. This $256 \times 256$ scene is down-sampled by factor $3 \times 3$ to $85 \times 85$ pixels and then interpolated to $255 \times 255$ by the various algorithms. The autocorrelation $\Phi_{s}$ of this scene is modeled by the asymmetric MRF with $d_{x}=7.618, d_{y}=4.550$, and $d_{c}=-0.023$ (by fitting the model to the autocorrelation function of the down-sampled image, as described in Appendix B). Fig. 8 illustrates: (a) the autocorrelation function of the original moon scene, (b) the MRF autocorrelation function that is fit to the autocorrelation function of the down-sampled image, and (c) the model error. As can be seen, the error from both modeling and fitting is relatively small (less than $5 \%$ of the peak). 


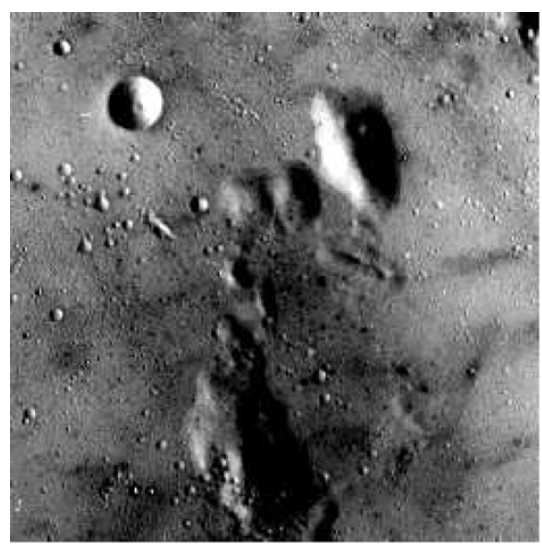

(a)

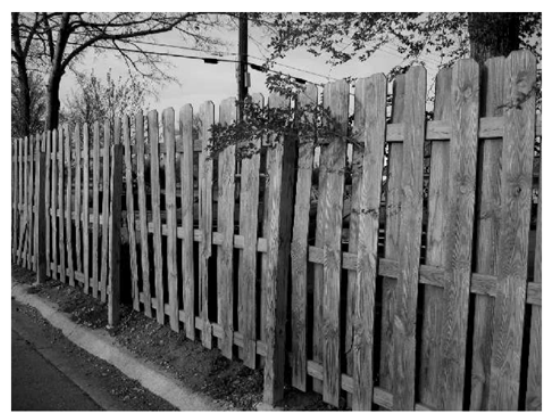

(b)

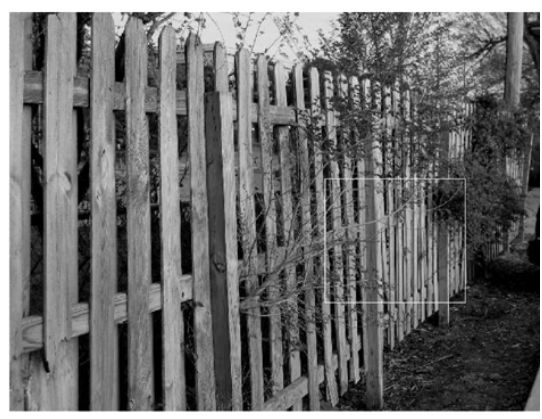

(c)

Fig. 7. Scenes used in the visual experiments. (a) Moon. (b) Fence1. (c) Fence2.

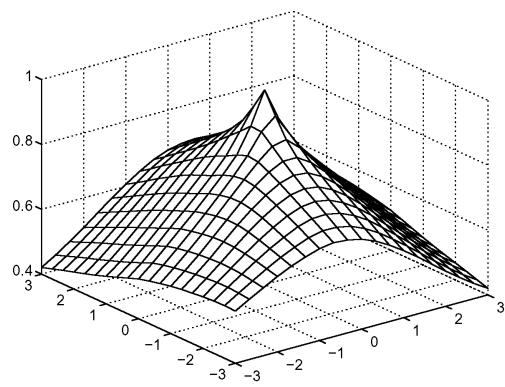

(a)

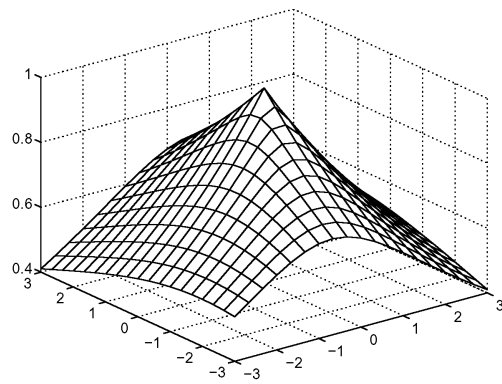

(b)

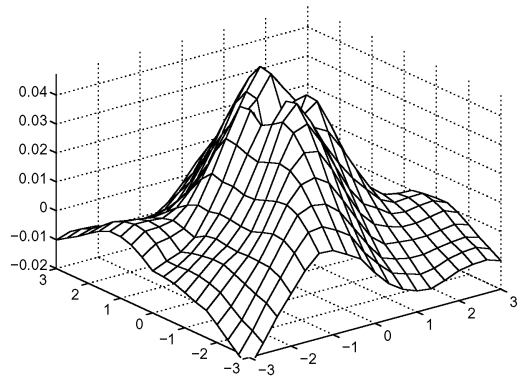

(c)

Fig. 8. Observed autocorrelation function of the moon scene, the MRF model approximation, and their difference. Only the relevant spatial support $([-3,3] \times$ $[-3,3]$ pixels) is graphed. (a) Actual $\Phi_{s a}$ and (b) $\operatorname{MRF} \Phi_{s m}$; (c) $\operatorname{MRF} \Phi_{s m}-\Phi_{s a}$.

With this model for the scene autocorrelation, the optimal parameter values for 2D-3PCC are $a_{33}=6.422, a_{32}=-8.811$, and $a_{30}=2.117$ and the optimal parameter values for 2D-5PCC are $a_{33}=6.767, a_{32}=-8.790, a_{23}=-9.630, a_{30}=1.950$, and $a_{03}=2.394$.

The interpolated images are shown in Fig. 9 (the results are not shown for edge-directed interpolation, because the zoom factor is not a power-of-two). To limit the boundary effects, the borders of all the processed images are cleared for visualization and are not included in the quantitative comparisons. The visual differences for most of the interpolated results are small, but artifacts related to aliasing are less evident with the unconstrained least-squares interpolator, 2D-3PCC, and 2D-5PCC interpolations. Table I lists the fidelity $\mathcal{F}$ of the various algorithms and various down-sampling factors $(2 \times 2,3 \times 3$, and $4 \times 4)$ for the moon scene. As expected, the unconstrained least-squares interpolator has the optimal fidelity. Of the constrained methods, 2D-5PCC has the highest fidelity. If the moon surface scene is down-sampled and interpolated by a factor of $2 \times 2$, there is less aliasing and the interpolation methods perform more similarly. However, if the down-sampling factor is $4 \times 4$, there is more aliasing and the benefit of parametric cubic convolution is more significant.

Table I also lists results for the same experimental procedure applied to six other scenes from the USC-SIPI database [24]: peppers, stream+bridge, tank, house+car, Lena, and man. All of these scenes are $512 \times 512$, except man, which is $1024 \times 1024$. In these also, the MRF model fits well and 2D-3PCC and
2D-5PCC perform well compared to other constrained filters. Cubic B-spline and separable bicubic convolution have slightly higher fidelity only for two images at $2 \times 2$ resampling (house+car and Lena) and for one image at $3 \times 3$ resampling (Lena). Edge-directed interpolation has slightly higher fidelity only for two images at $4 \times 4$ resampling (stream+bridge and tank). The slightly better performance of the separable cubic spline in the two images at $2 \times 2$ resampling evidences that the fitted autocorrelation model was suboptimal. Cubic B-spline interpolation performs relatively well only for lower resampling factors for which there is less aliasing and fidelity for all methods is very high (fidelity greater than 0.975). Conversely, edge-directed interpolation performs relatively well only for higher resampling factors where there is more aliasing associated with edges. Edge-directed interpolation is a more time-consuming adaptive filter and supports only power-of-two resampling factors. Two-dimensional cubic convolution performs relatively well across the various images and resampling factors-achieving the best fidelity in 15 of the 27 cases and identical fidelity (to three digits) in seven of the 27 cases. These results recommend 2-D cubic convolution for interpolating a wide range of images, if the autocorrelation function can be approximated and extra computation can be used (e.g., compared to separable bicubic convolution). The advantage of 2-D cubic convolution is greater for images with relatively finer details.

Parametric 2-D cubic convolution is an adaptive method and, in these experiments, is adapted on an image-by-image basis. Parametric 2-D cubic convolution could be used for adaptive 


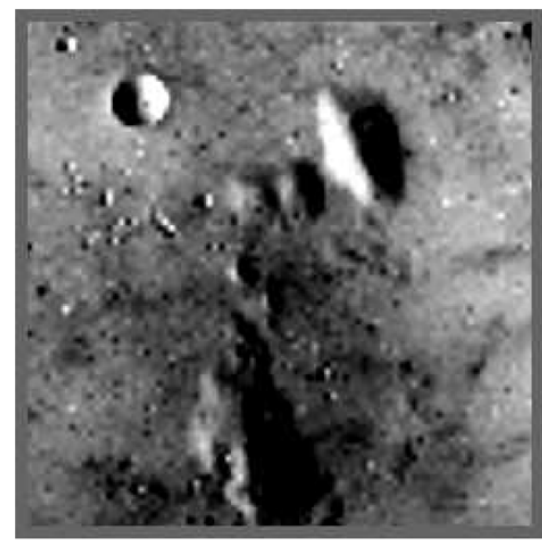

(a)

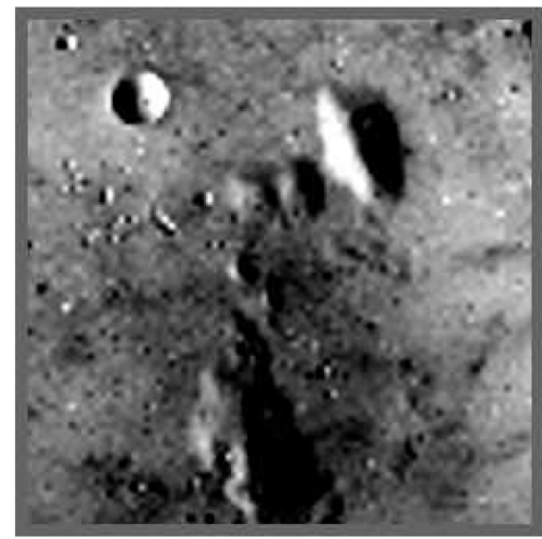

(d)

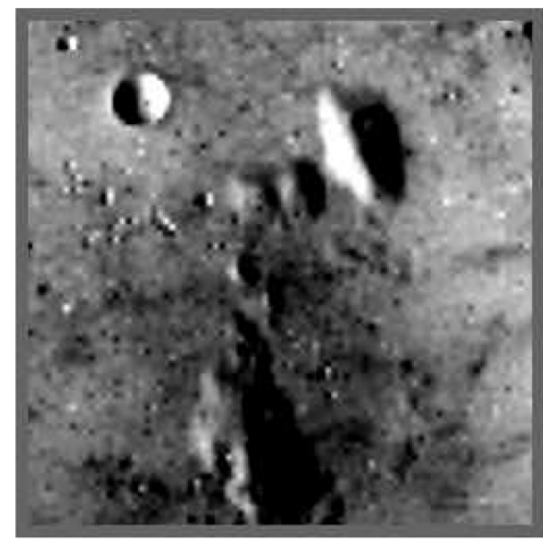

(b)

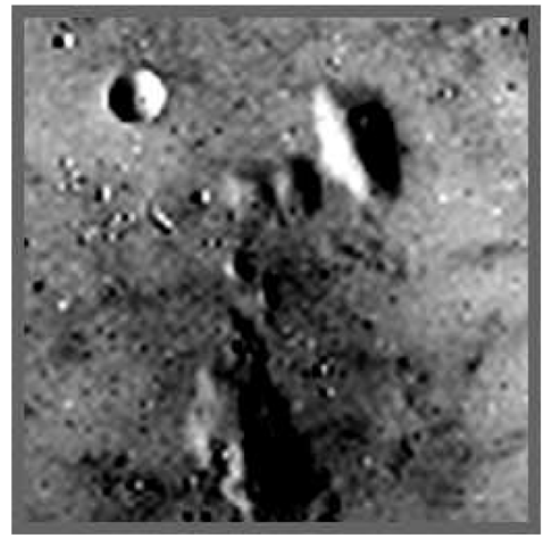

(e)

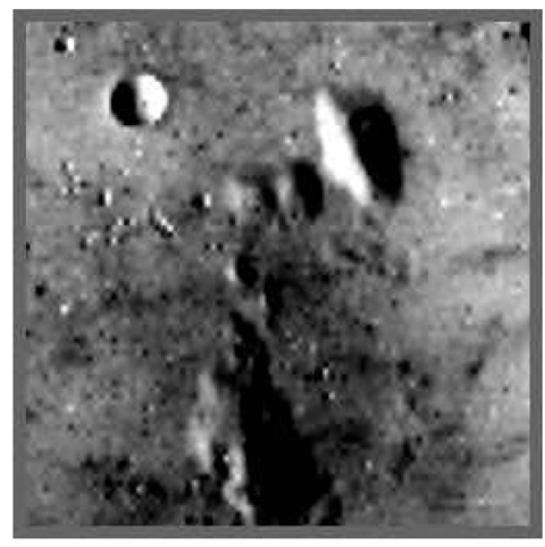

(c)

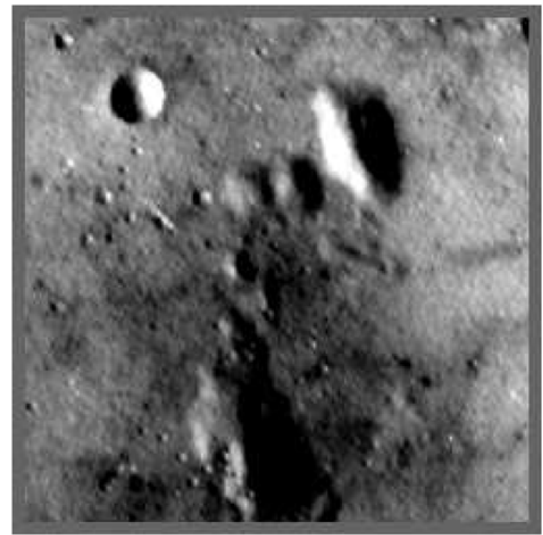

(f)

Fig. 9. The $256 \times 256$ moon surface scene is down-sampled to $85 \times 85$ and interpolated back to $255 \times 255$ by various algorithms. (a) $2 \mathrm{D}-2 \mathrm{PCC}$; (b) $2 \mathrm{D}-3 \mathrm{PCC}$; (c) 2D-5PCC; (d) separable bicubic; (e) cubic B-spline; and (f) optimal least-squares.

processing within an image by varying parameters based on local characterizations of the autocorrelation function.

\section{B. Scene Autocorrelation From Similar Images}

Fig. 7(b) and (c) presents two fence scenes acquired locally [the rectangle in Fig. 7(c) highlights a region to be expanded for visualization of the results]. Each $640 \times 480$ scene is down-sampled by factor $2 \times 2$ to $320 \times 240$ pixels and then interpolated to $640 \times 480$ by the various algorithms. In this experiment, the autocorrelation function of the $640 \times 480$ fence 1 scene is used as the estimate of the autocorrelation of fence 2 (and vice versa). Then, the optimal parameter values for 2D-3PCC are $a_{33}=$ 8.507, $a_{32}=-11.444$, and $a_{30}=2.705$ and the optimal parameter values for 2D-5PCC are $a_{33}=8.515, a_{32}=-11.279$, $a_{23}=-11.631, a_{30}=2.587$, and $a_{03}=2.824$.

The interpolated images for fence 2 are shown in Fig. 10. Again, the borders of all the processed images are cleared and excluded from the quantitative comparisons. Smaller regions of several of the images are presented in Fig. 11 to more clearly show differences. Visually, 2D-5PCC outperforms the other constrained methods. Table I lists the fidelity $\mathcal{F}$ of the various algorithms and various down-sampling factors. Again, the unconstrained least-squares interpolator is optimal, but 2D-5PCC has the highest fidelity of the constrained interpolators. Table I also lists results for the same experimental procedure applied to fence1, using the scene autocorrelation from fence2, and for various down-sampling and interpolation factors. Again, 2D-5PCC outperforms the other constrained interpolation methods, except for edge-directed interpolation applied to the fence 2 image resampled at $4 \times 4$. This example illustrates that the scene autocorrelation can be estimated by methods other than the MRF model described in the Appendices.

Table II lists the p-values of paired Student's t-tests for the fidelity in Table I. Each p-value is the probability that two interpolation methods have the same statistical mean fidelity for the same resampling density. The confidence level for performance differences between the optimal unconstrained least-squares interpolator and all other methods is high for each of the resampling factors. The confidence level for performance differences between 2D-5PCC and bicubic convolution is relatively good for $2 \times 2$ resampling (p-value 0.139 ), better for $3 \times 3$ resampling (p-value 0.059 ), and high for $4 \times 4$ resampling (p-value 0.030). The confidence levels for the differences between 2D-5PCC and cubic B-spline interpolation are uniformly higher than for bicubic convolution. The confidence levels for differences between 2D-5PCC and edge-directed interpolation are high for $2 \times 2$ resampling (where 2D-5PCC performs relatively well and edge-directed interpolation performs relatively poorly) and low for $4 \times 4$ resampling (where both methods 
TABLE I

Fidelity of VARIous Algorithms AND DOWN-SAMPling FACTORS FOR REAL SCENES. THE MRF SCENE PARAMETERS $d_{x}, d_{y}$, AND $d_{c}$ ARE ESTIMATED FROM The Down-SAmpled Images. A: Resampling Density $2 \times 2$. B: Resampling Density $3 \times 3$. C: Resampling Density $4 \times 4$

\begin{tabular}{|c|c|c|c|c|c|c|c|c|c|c|c|}
\hline Image & $d_{x}$ & $d_{y}$ & $d_{c}$ & $\frac{\left\|\bar{\Phi}_{p}-\Phi_{p}\right\|_{2}^{2}}{\left\|\bar{\Phi}_{p}\right\|_{2}^{2}}$ & $2 \mathrm{PCC}$ & $3 \mathrm{PCC}$ & $5 \mathrm{PCC}$ & Bicubic & B-spline & Edge-Dir. & Optimal \\
\hline moon & 10.626 & 6.718 & -0.010 & $6.203 \mathrm{E}-04$ & 0.931 & 0.932 & 0.932 & 0.929 & 0.924 & 0.928 & 0.948 \\
\hline peppers & 15.650 & 12.486 & 0.002 & $9.309 \mathrm{E}-05$ & 0.987 & 0.987 & 0.987 & 0.987 & 0.987 & 0.986 & 0.990 \\
\hline stream+bridge & 8.081 & 10.689 & -0.001 & $1.029 \mathrm{E}-03$ & 0.944 & 0.945 & 0.944 & 0.943 & 0.940 & 0.941 & 0.955 \\
\hline $\operatorname{tank}$ & 11.169 & 12.668 & 0.001 & $7.008 \mathrm{E}-04$ & 0.945 & 0.945 & 0.945 & 0.943 & 0.939 & 0.943 & 0.956 \\
\hline house + car & 9.914 & 8.443 & 0.000 & $4.589 \mathrm{E}-04$ & 0.977 & 0.977 & 0.977 & 0.978 & 0.978 & 0.973 & 0.981 \\
\hline lena & 22.260 & 10.803 & 0.002 & $3.740 \mathrm{E}-05$ & 0.990 & 0.990 & 0.990 & 0.992 & 0.992 & 0.988 & 0.994 \\
\hline man & 21.975 & 18.596 & 0.000 & $3.306 \mathrm{E}-05$ & 0.987 & 0.987 & 0.987 & 0.987 & 0.987 & 0.985 & 0.989 \\
\hline fence 1 & & & & & 0.835 & 0.836 & 0.836 & 0.821 & 0.809 & 0.830 & 0.874 \\
\hline fence 2 & & & & & 0.874 & 0.874 & 0.874 & 0.864 & 0.854 & 0.870 & 0.905 \\
\hline
\end{tabular}

B.

\begin{tabular}{|c||c|c|c|c||c|c|c||r|r|r||r|}
\hline Image & $d_{x}$ & $d_{y}$ & $d_{c}$ & $\frac{\left\|\bar{\Phi}_{p}-\Phi_{p}\right\|_{2}^{2}}{\left\|\bar{\Phi}_{p}\right\|_{2}^{2}}$ & 2 PCC & 3PCC & 5PCC & Bicubic & B-spline & Edge-Dir. & Optimal \\
\hline moon & 7.618 & 4.550 & -0.023 & $5.661 \mathrm{E}-04$ & 0.880 & 0.881 & 0.882 & 0.876 & 0.868 & \\
peppers & 9.986 & 7.808 & 0.003 & $2.116 \mathrm{E}-04$ & 0.976 & 0.977 & 0.977 & 0.976 & 0.975 & 0.908 \\
stream+bridge & 6.265 & 8.235 & -0.003 & $2.490 \mathrm{E}-03$ & 0.897 & 0.898 & 0.898 & 0.894 & 0.887 & 0.980 \\
tank & 8.666 & 9.899 & 0.001 & $1.397 \mathrm{E}-03$ & 0.903 & 0.904 & 0.904 & 0.900 & 0.894 & 0.915 \\
house+car & 6.797 & 6.777 & 0.000 & $1.524 \mathrm{E}-03$ & 0.944 & 0.944 & 0.944 & 0.944 & 0.941 & 0.922 \\
lena & 15.166 & 6.977 & 0.006 & $8.024 \mathrm{E}-05$ & 0.975 & 0.975 & 0.974 & 0.976 & 0.976 & 0.953 \\
man & 15.877 & 13.491 & 0.001 & $1.128 \mathrm{E}-04$ & 0.971 & 0.971 & 0.971 & 0.971 & 0.970 & 0.981 \\
fence1 & & & & & 0.740 & 0.743 & 0.743 & 0.725 & 0.709 & 0.976 \\
fence2 & & & & & 0.792 & 0.794 & 0.794 & 0.779 & 0.765 & \\
\end{tabular}

C.

\begin{tabular}{|c||c|c|c|c||c|c|c||r|r|r||r|}
\hline Image & $d_{x}$ & $d_{y}$ & $d_{c}$ & $\frac{\left\|\bar{\Phi}_{p}-\Phi_{p}\right\|_{2}^{2}}{\left\|\bar{\Phi}_{p}\right\|_{2}^{2}}$ & 2PCC & 3PCC & 5PCC & Bicubic & B-spline & Edge-Dir. & Optimal \\
\hline moon & 6.178 & 3.632 & -0.040 & $1.218 \mathrm{E}-03$ & 0.841 & 0.844 & 0.845 & 0.836 & 0.826 & 0.845 & 0.874 \\
peppers & 7.345 & 5.648 & 0.004 & $4.196 \mathrm{E}-04$ & 0.965 & 0.965 & 0.965 & 0.965 & 0.963 & 0.960 & 0.970 \\
stream+bridge & 5.350 & 6.913 & -0.005 & $4.516 \mathrm{E}-03$ & 0.859 & 0.860 & 0.860 & 0.854 & 0.844 & 0.862 & 0.884 \\
tank & 7.176 & 8.267 & 0.000 & $2.108 \mathrm{E}-03$ & 0.869 & 0.871 & 0.871 & 0.865 & 0.856 & 0.874 & 0.894 \\
house+car & 5.103 & 5.726 & 0.000 & $3.277 \mathrm{E}-03$ & 0.910 & 0.911 & 0.911 & 0.909 & 0.904 & 0.906 & 0.924 \\
lena & 11.541 & 5.119 & 0.012 & $2.545 \mathrm{E}-04$ & 0.955 & 0.955 & 0.955 & 0.955 & 0.953 & 0.951 & 0.964 \\
man & 12.687 & 10.880 & 0.002 & $2.254 \mathrm{E}-04$ & 0.954 & 0.954 & 0.954 & 0.953 & 0.951 & 0.952 & 0.961 \\
fence1 & & & & & 0.678 & 0.681 & 0.682 & 0.660 & 0.641 & 0.681 & 0.742 \\
fence2 & & & & & 0.738 & 0.741 & 0.742 & 0.723 & 0.707 & 0.746 & 0.794 \\
\hline
\end{tabular}

perform relatively well). These results support the conclusion that there are performance differences for 2D-5PCC, especially for interpolation of finer details.

\section{CONCLUSION}

Separable bicubic convolution is one of the most popular methods for image interpolation, but it is based on the assumption of separability, which typically is not true. This paper examines the theoretical and practical issues of nonseparable, 2-D cubic convolution.

This paper develops two nonseparable, 2-D cubic-convolution kernels. The first kernel, 2D-3PCC, has three parameters and is the most general 2-D, piecewise-cubic interpolator defined on $[-2,2] \times[-2,2]$ with constraints for biaxial symmetry, diagonal (or $90^{\circ}$ rotational) symmetry, continuity, and smoothness. The second kernel, 2D-5PCC, relaxes the diagonal-symmetry constraint on 2D-3PCC and thus is more general. The roadmap of relaxing constraints could be used to develop other formulations of cubic convolution.

This paper also develops a closed-form solution for the optimal parameters that minimize the expected MSE over an ensemble of scenes characterized by autocorrelation. The spatialdomain formulation for optimal parameterization is computationally efficient and establishes the basis for locally adaptive filters. The closed-form solution supports any approximation of scene autocorrelation and experimental results show that parameterization is robust with respect to approximation errors. In particular, this paper presents a MRF model with affine transformation to model scene autocorrelation and derives a closed-form solution to fit the model. Experimental results show that, for a 


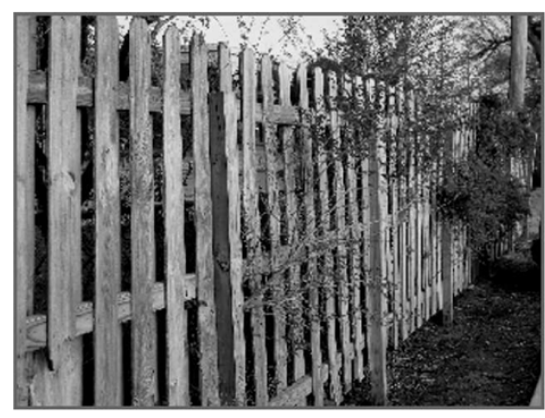

(a)

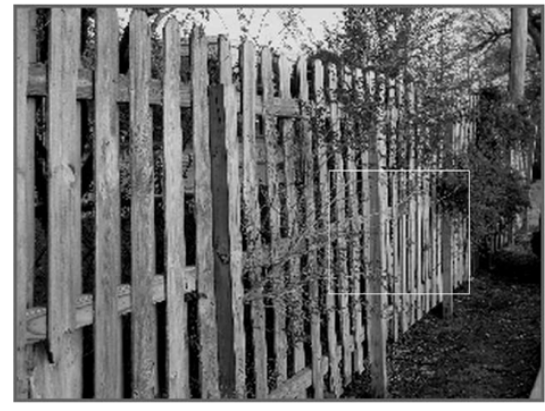

(d)

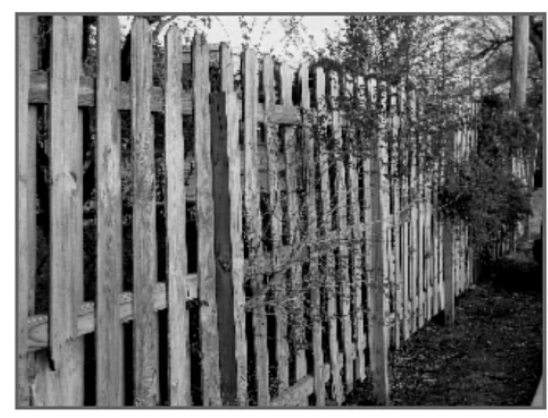

(b)

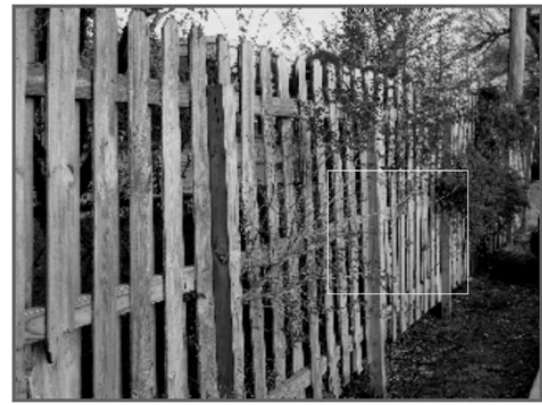

(e)

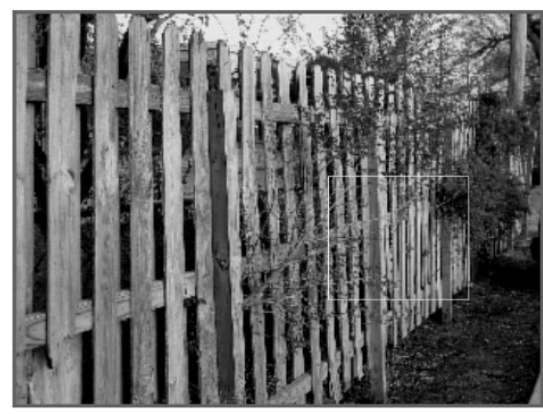

(c)

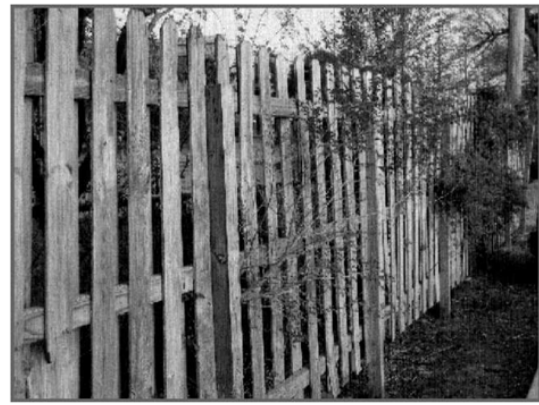

(f)

Fig. 10. The $640 \times 480$ fence 2 scene is down-sampled to $320 \times 240$ and interpolated back to $640 \times 480$ by various algorithms. (a) Separable bicubic; (b) 2D-3PCC; (c) 2D-5PCC; (d) cubic B-spline; (e) edge-directed; (f) optimal least-squares.

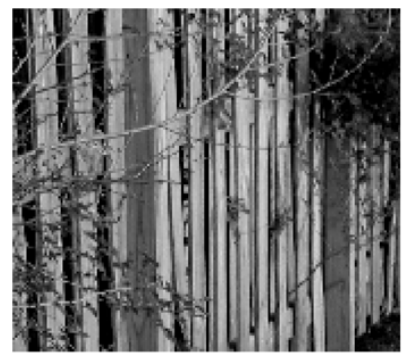

(a)

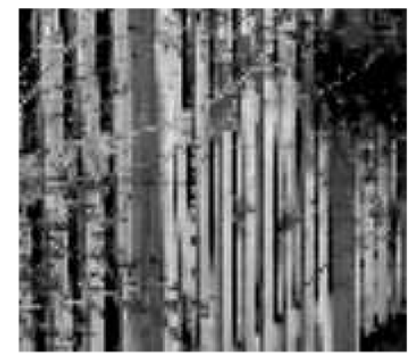

(b)

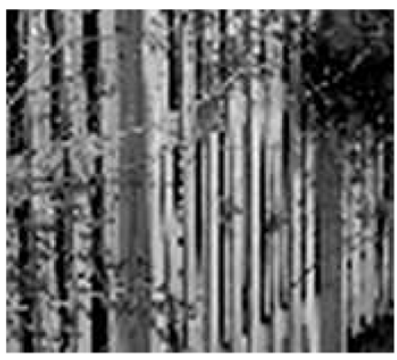

(c)

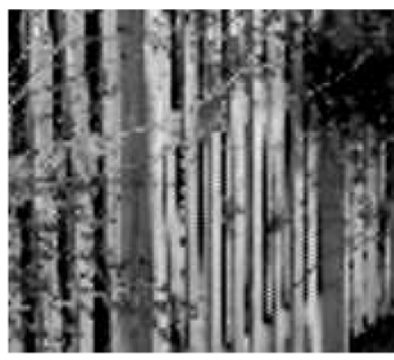

(d)

Fig. 11. Images (a)-(d) are cropped, respectively, from Figs 7(c) and 10(c)-(e). (a) Portion of fence2; (b) 2D-5PCC; (c) cubic B-spline; and (d) edge-directed.

range of scenes, the model can be fit to closely estimate the scene autocorrelation using only the autocorrelation of a down-sampled image. The closed-form solution for the model fit establishes the basis for locally adaptive characterization of the scene autocorrelation.

Quantitative fidelity analyses and visual experiments indicate that 2D-3PCC and 2D-5PCC outperform several popular interpolation methods (including a spatially adaptive interpolator) for real scenes. In theoretical and experimental analyses, both 2D-3PCC and 2D-5PCC better balance error budget tradeoffs involving scene signal and aliasing and more closely resemble the unconstrained least-squares interpolator over a range of scenes with different spatial content. In these analyses, 2D-5PCC is especially effective compared to separable methods for interpolating off-axes spatial frequencies. Both 2D-3PCC and 2D-5PCC are low-order polynomials with small spatial support and so are easy to implement and efficient to apply in the spatial domain, but have higher computational cost than separable kernels.

Our ongoing research is extending nonseparable cubic convolution to image restoration and exploiting other random field models. The 2D-3PCC and 2D-5PCC kernels are derived subject to several constraints related to interpolation: the kernel value at $(0,0)$ is constrained to one and the values at the other knots are constrained to zero. Relaxing these constraints allows the kernels to correct for acquisition artifacts and perform image restoration [25], [26]. Parametric cubic convolution can be optimized for any random field model. The geostatistical community uses random fields widely. It is recognized that properties of optimal interpolation kernels are determined mainly by the local behavior of the semivariogram of a presumed random field [27]. Both 2D-3PCC and 2D-5PCC are designed to exploit local autocorrelation and so are candidates for efficient interpolation with respect to random fields. 
TABLE II

P-VAlues of Paired Student's T-Tests For the Fidelity in TABle I. A: ESAMPLING DENSITY $2 \times 2$. B: RESAMPLING DENSITY $3 \times 3$. C: RESAMPLING DENSITY $4 \times 4$

\begin{tabular}{|r|c|c|c|c|c|c|}
\multicolumn{7}{|c}{ A. } \\
\hline 3PCC & 2PCC & 3PCC & 5PCC & Bicubic & B-spline & Edge-Dir. \\
5PCC & 0.107 & 0.504 & & & & \\
Bicubic & 0.152 & 0.138 & 0.139 & & & \\
B-spline & 0.073 & 0.070 & 0.072 & 0.032 & & \\
Edge-Dir. & 0.000 & 0.000 & 0.000 & 0.852 & 0.261 & \\
Optimal & 0.015 & 0.015 & 0.015 & 0.029 & 0.029 & 0.008 \\
\hline
\end{tabular}

\begin{tabular}{|r|c|c|c|c|c|}
\hline & 2PCC & 3PCC & 5PCC & Bicubic & B-spline \\
\hline 3PCC & 0.037 & & & & \\
5PCC & 0.049 & 0.325 & & & \\
Bicubic & 0.067 & 0.059 & 0.059 & & \\
B-spline & 0.024 & 0.024 & 0.024 & 0.009 & \\
Optimal & 0.012 & 0.011 & 0.011 & 0.017 & 0.015 \\
\hline
\end{tabular}

C.

\begin{tabular}{|r|c|c|c|c|c|c|}
\hline & 2PCC & 3PCC & 5PCC & Bicubic & B-spline & Edge-Dir. \\
\hline 3PCC & 0.007 & & & & & \\
5PCC & 0.012 & 0.147 & & & & \\
Bicubic & 0.042 & 0.029 & 0.030 & & & \\
B-spline & 0.012 & 0.011 & 0.011 & 0.003 & & \\
Edge-Dir. & 0.657 & 0.565 & 0.449 & 0.122 & 0.031 & \\
Optimal & 0.006 & 0.006 & 0.006 & 0.009 & 0.008 & 0.007 \\
\hline
\end{tabular}

\section{APPENDIX}

\section{A. Markov Random Field}

The autocorrelation function $\Phi_{s}$ of an isotropic Markov random field (MRF) is an appropriate model for many ensembles of both images and geo-statistical quantities [28]

$$
\Phi_{s}(\tau)=e^{-\frac{|\tau|}{d}}, \quad d>0
$$

where $\tau=\sqrt{x^{2}+y^{2}}$ and $d$ is the mean spatial detail (MSD). The MSD can be interpreted as the average size of details in the scene along a line in any direction. Scenes with larger objects (relative to the sampling interval) have larger MSD. 2D-3PCC is appropriate for this model because the model is nonseparable and has biaxial and diagonal symmetry.

A more general autocorrelation model without rotational symmetry can be generated by affine transformation (without translation) of the isotropic MRF, which introduces three parameters

$\Phi_{s}(x, y)=e^{-\sqrt{\frac{x^{2}}{d_{x}^{2}}+\frac{y^{2}}{d_{y}^{2}}+d_{c} x y}}, \quad d_{x}>0, d_{y}>0,\left|d_{c}\right| \leq \frac{2}{d_{x} d_{y}}$.

For $d_{c}=0, d_{x}$ and $d_{y}$ can be understood as the mean spatial details along the $x$ axis and $y$ axis, respectively. The parameter $d_{c}$ allows rotational orientation. 2D-5PCC is appropriate for this model because the model is rotationally asymmetric. Translational parameters could be added to create a nonhomogeneous (or nonstationary) model for adaptive processing.

\section{B. Autocorrelation Estimation Based on MRF}

As detailed in Section III-A, the optimal parameters for cubic convolution can be determined for any given scene autocorrelation function $\Phi_{s}$. However, the scene autocorrelation function typically is unknown and must be estimated. One approach for estimating the scene autocorrelation function is to compute the autocorrelation function from one or more high-resolution images of a similar scene(s). Another approach is to fit a mathematical scene model to the autocorrelation function observed in the image. This section describes fitting the MRF scene model presented in Appendix A. A similar approach could be used for any scene model.

The scene autocorrelation function is a continuous function that can be observed in the image only at discrete intervals on the sampling grid. The MRF autocorrelation function in (28) can be fit to the observed image autocorrelation function by iterative numerical methods, but a closed-form fit is possible if the model is linearized in terms of its three parameters. First, (28) can be rewritten as

$$
\Phi_{s}(x, y)=e^{-\sqrt{a x^{2}+b y^{2}+c x y}}
$$

where $a=1 / d_{x}^{2}, b=1 / d_{y}^{2}$, and $c=d_{c}$. Given the observed autocorrelation function of the image $\bar{\Phi}_{p}$, the expected mean square error (MSE) of the linearized fit at the pixel intervals is

$$
\begin{aligned}
& J(a, b, c) \\
& =\sum_{m=-3}^{3} \sum_{n=-3}^{3}\left|\log ^{2} \bar{\Phi}_{p}[m, n]-\log ^{2} \Phi_{s}(m, n)\right|^{2} \\
& =\sum_{m=-3}^{3} \sum_{n=-3}^{3}\left|\log ^{2} \bar{\Phi}_{p}[m, n]-\left(a m^{2}+b n^{2}+c m n\right)\right|^{2} .
\end{aligned}
$$

Computing the partial derivatives of $J$ with respect to $a, b$, and $c$, and solving for the simultaneous equality with zero yields a model fit

$$
\begin{aligned}
a & =\frac{1}{1267728} \sum_{m=-3}^{3} \sum_{n=-3}^{3}\left(1372 m^{2}-784 n^{2}\right) \log ^{2} \bar{\Phi}_{p}[m, n] \\
b & =\frac{1}{1267728} \sum_{m=-3}^{3} \sum_{n=-3}^{3}\left(1372 n^{2}-784 m^{2}\right) \log ^{2} \bar{\Phi}_{p}[m, n] \\
c & =\frac{1}{784} \sum_{m=-3}^{3} \sum_{n=-3}^{3} m n \log ^{2} \bar{\Phi}_{p}[m, n] .
\end{aligned}
$$

The equations in (31) can be computed globally for a global filter or locally for an adaptive filter.

\section{REFERENCES}

[1] T. M. Lehmann, C. Gonner, and K. Spitzer, "Survey: Interpolation methods in medical image processing," IEEE Trans. Med. Imag., vol. 18, no. 11, pp. 1049-1075, Nov. 1999.

[2] E. Meijering, "A chronology of interpolation: From ancient astronomy to modern signal and image processing," Proc. IEEE, vol. 90, no. 3, pp. 319-342, Mar. 2002 
[3] S. S. Rifman, "Digital rectification of ERTS multispectral imagery," in Proc. Symp. Significant Results Obtained From ERTS-1, vol. I, sec. B. NASA SP-327, 1973, pp. 1131-1142.

[4] R. G. Keys, "Cubic convolution interpolation for digital image processing," IEEE Trans. Acoust., Speech, Signal Process., vol. ASSP-29, no. 6, pp. 1153-1160, Dec. 1981.

[5] S. K. Park and R. A. Schowengerdt, "Image reconstruction by parametric cubic convolution," Comput. Vis., Graph., Image Process., vol. 23, pp. 258-272, 1983.

[6] S. E. Reichenbach and F. Geng, "Two-dimensional cubic convolution," IEEE Trans. Image Process., vol. 12, no. 8, pp. 857-865, Aug. 2003.

[7] - "Improved cubic convolution for two-dimensional image reconstruction," in Proc. IEEE Nuclear Science Symp., Medical Imaging Conf., 2001, pp. 1775-1778.

[8] T. Blu, P. Thévenaz, and M. Unser, "Linear interpolation revitalized," IEEE Trans. Image Process., vol. 13, no. 5, pp. 710-719, May 2004.

[9] E. H. W. Meijering, K. J. Zuiderveld, and M. A. Viergever, "Image reconstruction by convolution with symmetrical piecewise nth-order polynomial kernels," IEEE Trans. Image Process., vol. 8, no. 2, pp. 192-201, Feb. 1999.

[10] P. Thévenaz, T. Blu, and M. Unser, "Interpolation revisited," IEEE Trans. Med. Imag., vol. 19, no. 7, pp. 739-758, Jul. 2000.

[11] D. D. Muresan and T. W. Parks, "Adaptively quadratic (aqua) image interpolation," IEEE Trans. Image Process., vol. 13, no. 5, pp. 690-698, May 2004.

[12] K. R. Castleman, Digital Image Processing. Englewood Cliffs, NJ: Prentice-Hall, 1996.

[13] C. L. Fales, F. O. Huck, J. A. McCormick, and S. K. Park, "Wiener restoration of sampled image data: End-to-end analysis," J. Opt. Soc. Amer. A, vol. 5, no. 3, pp. 300-314, 1988.

[14] W. Cheney and W. Light, A Course in Approximation Theory. Pacific Grove, CA: Brooks/Cole, 2000.

[15] A. K. Jain, Fundamentals of Digital Image Processing. Englewood Cliffs, NJ: Prentice-Hall, 1989.

[16] E. H. Linfoot, "Transmission factors and optical design," J. Opt. Soc. Amer., vol. 46, no. 9, pp. 740-752, 1956.

[17] M. Unser, "Splines: a perfect fit for signal and image processing," IEEE Signal Process. Mag., vol. 16, no. 6, pp. 22-38, Jun. 1999.

[18] T. Blu, P. Thévenaz, and M. Unser, "MOMS: Maximal-order interpolation of minimal support," IEEE Trans. Image Process., vol. 10, no. 7, pp. 1069-1080, Jul. 2001.

[19] S. K. Park, "Image gathering, interpolation and restoration: A fidelity analysis," Proc. SPIE Visual Information Processing, vol. 1705, pp. 134-144, 1992.

[20] P. Thévenaz, T. Blu, and M. Unser, Image Interpolation and Resampling, I. Bankman, Ed. San Diego, CA: Academic, 2000.

[21] E. H. W. Meijering, W. J. Niessen, and M. A. Viergever, "Quantitative evaluation of convolution-based methods for medical image interpolation," Med. Image Anal., vol. 5, no. 2, pp. 111-126, 2001.

[22] X. Li and M. T. Orchard, "New edge-directed interpolation," IEEE Trans. Image Process., vol. 10, no. 10, pp. 1521-1527, Oct. 2001

[23] — , New edge-directed interpolation. [Online]. Available: http://www.csee.wvu.edu/xinl/code/nedi.zip, 2005.
[24] A. Weber, USC-SIPI Image Database, Univ. Southern California. [Online]. Available: http://sipi.usc.edu/services/database, 2004.

[25] S. E. Reichenbach and S. K. Park, "Two-parameter cubic convolution for image reconstruction," Proc. SPIE, vol. 1199, pp. 833-840, 1989.

[26] S. E. Reichenbach and K. Haake, "Cubic convolution for one-pass restoration and resampling," in Proc. IEEE Int. Geoscience Remote Sensing Symp., vol. III, 1996, pp. 1597-1599.

[27] M. L. Stein, Interpolation of Spatial Data: Some Theory for Kriging. New York: Springer-Verlag, 1999.

[28] R. A. Schowengerdt, Remote Sensing: Models and Methods for Image Processing, 2nd ed. Orlando, FL: Academic, 1997.

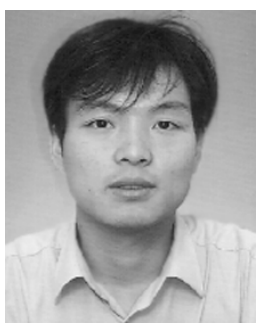

Jiazheng Shi received the B.E. and M.E. degrees in electrical engineering from the Beijing University of Posts and Telecommunications, Beijing, China, in 1997 and 2000, respectively, and the Ph.D. degree in computer science from the University of Nebraska, Lincoln, in 2005.

In 2000, he was with Global Software Group, Motorola, Inc. Currently, he is a Postdoctoral Research Fellow with the Computer-Aided Diagnosis Laboratory, Department of Radiology, University of Michigan, Ann Arbor. His research interests are image processing, computer vision, and robotics.

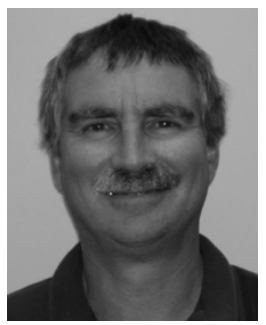

Stephen E. Reichenbach received the B.A. degree in English from the University of Nebraska, Lincoln (UNL), in 1976, the M.S. degree in computer science from Washington University, St. Louis, MO, in 1985, and Ph.D. degree in computer science from the College of William and Mary, Williamsburg, VA, in 1989.

He is a Professor with the Computer Science and Engineering (CSE) Department, UNL, where he has been on the faculty since 1990 and served as Department Chairman from 1996 to 2000 . He held a National Research Council research associateship in the Visual Information Processing Laboratory at the NASA Langley Research Center, and an ASEE research fellowship in the Landsat 7 Project Science Office. He has authored more than 100 technical articles on digital image processing and image information systems. In 1994, he founded Internet Nebraska Corporation, the oldest and largest Nebraska-based Internet service provider. In 2003, he founded GC Image, LLC, to develop advanced informatics software for comprehensive two-dimensional gas chromatography (GCxGC), a powerful new technology for chemical separations. 